\title{
PBL Planner Online: Uma ferramenta colaborativa para o planejamento de ensino na abordagem PBL em cursos de Computação
}

\author{
Jesiel Viana da Silva ${ }^{1,2}$, Gustavo Henrique da Silva Alexandre ${ }^{1}$ \\ ${ }^{1}$ Cesar School - Recife, PE - Brazil \\ ${ }^{2}$ Instituto Federal do Piauí (IFPI) - Picos, PI - Brazil \\ jesiel@ifpi.edu.br, gugahenrique@gmail.com
}

\begin{abstract}
Teaching planning in the Problem-Based Learning (PBL) approach is not a simple task, due to the number of aspects involved and the lack of specific tools. In this context, this paper presents a virtual tool to support collaborative planning in the PBL approach in the teaching of Computing, called PBL Planner Online. It is adherent to PBL principles and allows distributed collaboration in a synchronous and asynchronous way. The validation included the evaluation of usability and acceptance of the proposed tool, as well as its use in real environments for collaborative planning performed in a distributed way. The analyzed results indicated good usability and acceptance of the tool by teachers to carry out distributed collaborative planning.
\end{abstract}

Resumo. O planejamento de ensino na abordagem Problem-Based Learning (PBL) não é uma tarefa simples, devido a quantidade de aspectos envolvidos e a falta de ferramentas específicas. Nesse contexto, este trabalho apresenta uma ferramenta para apoiar o planejamento colaborativo na abordagem PBL no ensino de Computação, chamada de PBL Planner Online. Ela é aderente aos princípios PBL e permite a colaboração distribuída de forma síncrona e assíncrona. A validação compreendeu a avaliação de usabilidade e aceitação da ferramenta proposta, bem como sua utilização em ambientes reais para planejamento colaborativo realizado de forma distribuída. Os resultados analisados indicaram boa usabilidade e aceitação da ferramenta pelos professores para realização do planejamento colaborativo distribuído.

\section{Introdução}

O método de ensino Problem-Based Learning (PBL) tem mostrado-se bastante satisfatório no ensino de Computação, promovendo uma melhoria na formação profissional dos estudantes, através de uma abordagem de ensino que trabalha com problemas reais da área [OLIVEIRA; SANTOS; GARCIA, 2013].

Apesar dos benefícios do método PBL, estudos realizados por Santos et al. (2018) indicam que sua utilização não é simples. Os principais desafios para adoção do PBL estão relacionados ao planejamento e gerenciamento do processo de ensino e aprendizagem, visto que o PBL demanda transformações no âmbito educacional para que este possa refletir a realidade do ambiente profissional. [RODRIGUES; SANTOS, 2016]. 
Segundo Oliveira, Santos e Garcia (2013), o desconhecimento sobre os fundamentos metodológicos do PBL e a falta de planejamento adequado são os principais fatores que impactam negativamente na aplicação do método. De acordo com Santos et al. (2018), a efetividade do PBL está diretamente ligada a dois fatores: (i) aderência aos princípios PBL [SANTOS; FIGUERÊDO; WANDERLEY, 2013] e (ii) o uso de ferramentas de gestão que permitam o planejamento, execução e acompanhamento do PBL ao longo de sua adoção.

A participação e colaboração de todos os envolvidos no processo de ensino durante o planejamento é fundamental para evitar que aspectos vitais do método PBL sejam negligenciados ou esquecidos [ALEXANDRE, 2018]. De acordo com Baniya et al. (2019), a comunicação e colaboração ativa entre docentes são fatores essenciais para o sucesso do processo de ensino multidisciplinar.

Devido à característica multidisciplinar do PBL [SAVERY, 2015], que envolve professores de diferentes disciplinas e um conjunto de variáveis a serem consideradas, fica evidente que a adoção do PBL não é uma tarefa fácil e o risco do método não ser efetivo durante sua execução é alto, caso não seja bem planejado e acompanhado [ALEXANDRE, 2018]. Portanto, para adoção do PBL, é de suma importância a utilização de ferramentas que auxiliem em seu planejamento, aplicação e monitoramento, permitindo a colaboração de membros remotos de forma síncrona e assíncrona e, assim, proporcionar flexibilidade temporal e geográfica para realização de trabalho colaborativo [ALEXANDRE et al., 2018].

Para atender as carências expostas acima, este artigo propõe o PBL Planner Online $(P P O)^{1}$, uma ferramenta colaborativa aderente aos princípios PBL, que permite a colaboração distribuída entre educadores, automatiza a geração de artefatos, possibilita a gestão de tarefas e, por fim, facilita o compartilhamento de exemplos. Ela é voltada para o planejamento PBL em cursos de Computação, baseada no PBL Toolkit.

\section{Fundamentação Teórica}

\subsection{Problem-Based Learning - PBL}

PBL é um método de ensino construtivista centrado no aluno que utiliza problemas reais como objeto de aprendizagem, onde o aluno desenvolve a capacidade de aplicar conhecimentos técnicos e habilidades interpessoais para resolução dos problemas, por meio do trabalho em equipe, visão crítica e reflexão do processo de aprendizagem [SAVERY, 2015].

De acordo com Savery (2015), o método PBL é baseado em princípios. A garantia desses princípios exige um alto investimento na aplicação e gestão do PBL, que inclui tempo, esforço, recursos e processos de controle e monitoramento [SANTOS, 2016]. Aderência aos seus princípios, gerenciamento dos processos de planejamento e execução ao longo dos ciclos de aprendizado são essenciais para eficácia da abordagem PBL, portanto, não podem ser descartados [SANTOS et al., 2018].

Quanto aos desafios no processo de ensino e aprendizagem com PBL na área de Computação, a fase de planejamento educacional é um fator chave para obtenção de bons

\footnotetext{
${ }^{1}$ https://pblplanner.jesielviana.com
} 
resultados [OLIVEIRA; SANTOS; GARCIA, 2013]. Segundo Gomides et al. (2019), o planejamento interdisciplinar necessário e constante no desenvolvimento adequado de um currículo PBL é uma das grandes dificuldades da sua execução.

Para realização do planejamento interdisciplinar faz-se necessário a colaboração. De acordo com Assis e Almeida (2017), o planejamento de ensino colaborativo inclui a atuação docente entre pares nas ações da prática pedagógica, fazendo junto o planejamento e também compartilhando exemplos do que deu certo e o que deu errado.

\subsection{Sistemas Colaborativos}

Conceitualmente, os sistemas computacionais com um ambiente compartilhado projetado para grupos de pessoas colaborarem em um objetivo comum são conhecidos como sistemas colaborativos (groupware em inglês)[RAMA; BISHOP, 2006]. Eles buscam diminuir as barreiras impostas pelo espaço físico e o tempo para promover a comunicação e ampliar a colaboração, otimizando os processos de tomada de decisão em grupo [BROUGHTON et al., 2009].

Segundo Ellis, Gibbs e Rein (1991), groupware permite que usuários interajam com outros usuários de forma comunicativa, coordenada e cooperativa para realização de tarefas com objetivo comum por meio de um ambiente virtual compartilhado. Baseado nestes conceitos, [FUKS et al., 2004] apresentam o Modelo 3C de Colaboração formado pelas dimensões comunicação, coordenação e cooperação.

O modelo 3C de colaboração posiciona cada $\mathrm{C}$, ou dimensão, numa extremidade, mas, apesar da separação com o propósito de análise, as dimensões comunicação, coordenação e cooperação não devem ser vistas de maneira isolada, pois são interdependentes [PIMENTEL et al., 2006].

A percepção também é um conceito essencial no modelo $3 \mathrm{C}$, que permeia os $3 \mathrm{Cs}$ [FUKS et al., 2003]. Ela conscientiza o usuário de que ele faz parte de um grupo, permitindo que ele perceba o que está acontecendo, o que as outras pessoas estão fazendo e também adquire informações necessárias para seu trabalho [FUKS et al., 2003].

\section{Trabalhos Relacionados}

No contexto da adoção do PBL em cursos da área de Computação, algumas ferramentas já foram propostas para a etapa de planejamento, tais como o PBL-Tutor Canvas [VIDAL; SANTOS; CARVALHO, 2016] e PBL Planner Toolkit (PBL Toolkit) [ALEXANDRE, 2018]. Elas são ferramentas visuais e colaborativas, no entanto, permitem somente a colaboração presencial, não possuindo recursos para participação de membros remotos.

O PBL Toolkit tem como objetivo apoiar o planejamento de ensino na abordagem PBL em cursos de Computação. É composto por dois elementos principais: PBL Canvas e Cartas PBL.

O PBL Canvas tem o formato de um quadro dividido em 11 (onze) campos. A definição dos campos que formam o PBL Canvas originaram-se da metodologia xPBL [SANTOS; FURTADO; LINS, 2014]. Cada campo representa um conjunto de aspectos relacionados ao planejamento.

Já as Cartas PBL são um conjunto de 40 (quarenta) cartas distribuídas entre os 
11 (onze) campos do PBL Canvas. O objetivo das cartas é guiar o preenchimento do planejamento [ALEXANDRE, 2018].

Embora as ferramentas existentes auxiliem no planejamento de ensino PBL, há carências que dificultam o processo de planejamento e a adoção do método. Uma dessas carências é a falta de suporte à colaboração distribuída [ASSIS; ALMEIDA, 2017]. Também há restrições quanto à flexibilidade de acesso e operacionalização das ferramentas, além da necessidade de recursos de monitoramento e controle [ALEXANDRE et al., 2018].

Outro ponto a se destacar nas ferramentas atuais é a dificuldade de compartilhar exemplos de planejamentos já realizados que ajudariam quanto ao preenchimento de novos planejamentos por iniciantes no método PBL [ALEXANDRE, 2018].

\section{PBL Planner Online (PPO)}

A metodologia utilizada neste trabalho é baseada na aplicação do método Design Science Research (DSR) descrito por Wieringa (2014). DSR é indicado para pesquisas de cunho tecnológico que envolve a compreensão de um problema, construção e avaliação de artefatos [BARBOSA; BAX, 2017]. Segundo Wieringa (2014), DSR é uma abordagem científica para construção e investigação de artefatos em um determinado contexto.

Para desenvolvimento do $P P O$ foram executados dois ciclos $D S R$ proposto por Wieringa (2014), que é composto pelas seguintes etapas: Investigação do problema, Design da Solução e Validação da Solução, Implementação da Solução e Avaliação da Implementação. As três primeiras etapas são voltadas para a concepção e construção da solução, no qual seu resultado - uma proposta de solução validada - é o artefato de entrada para as outras duas etapas, que têm como objetivo aplicar e avaliar a solução em um ambiente real.

O PPO é um sistema Web colaborativo para auxiliar o planejamento de ensino na abordagem PBL em cursos de Computação alinhado aos princípios do método PBL e com suporte à colaboração distribuída de forma síncrona e assíncrona. Sua concepção teve como base os elementos e o processo de planejamento do PBL Toolkit.

O PPO busca diminuir as barreiras impostas pelo espaço físico e o tempo para facilitar a colaboração possibilitando flexibilidade temporal e geográfica para realização de trabalho em grupo. Além disso, tem o propósito de automatizar algumas etapas do processo de planejamento.

\subsection{Arquitetura}

O PPO foi estruturado de acordo com o modelo cliente-servidor, uma arquitetura de aplicação distribuída em que o servidor atua como um provedor de recursos e serviços que são requeridos pelos clientes [BERTOCCO et al., 1998]. A arquitetura distribuída é a mais usada em groupware [GOMES; WILLRICH; RIVERA, 2011]

O PPO foi construído baseado nas características da arquitetura centralizada e descentralizada, possuindo um servidor central responsável pelo controle dos dados e gerenciamento do ambiente de trabalho compartilhado e os clientes que gerenciam o espaço de trabalho privado de cada usuário e realiza todo o processamento gráfico. 
A Figura 1 apresenta o diagrama de arquitetura de software do $P P O$, essa representação foi baseada no diagrama de contexto do modelo C4 [BROWN, 2018], que mostra uma visão geral do sistema e como ele se encaixa no mundo real em relação aos seus usuários.

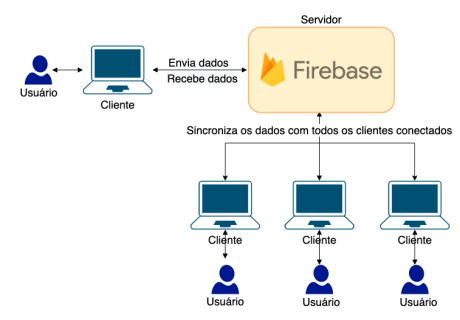

Figura 1. Visão geral da arquitetura

O diagrama de contexto do $P P O$ (Figura 1), apresenta os seguintes elementos: os usuários que acessam o sistema para realizar alguma operação, o servidor central responsável por fazer toda a gestão dos dados e sincronização do ambiente virtual compartilhado e os aplicativos clientes que são responsáveis pelo controle e renderização da interface gráfica para os usuários do sistema.

O modelo arquitetural do $P P O$ permite a separação em camadas dos componentes responsáveis pela interação com o usuário (cliente) e os componentes responsáveis pelo processamento e gerenciamento dos dados (servidor). A seguir serão explanadas cada camada detalhadamente.

A Camada Cliente é a responsável por possibilitar a interação dos usuários com o sistema por meio de uma interface Web. Para construção da Camada Cliente, além das tecnologias básicas da Web (HTML, CSS e Javascript) foram utilizados dois frameworks: Vue.js para desenvolvimento da interface visual e o Firebase JavaScript(JS) SDK que fornece a infraestrutura e API necessária para comunicação com a Camada Servidor.

A Camada Servidor é responsável pelo controle de acesso ao sistema, bem como por todas as regras de negócios dos requisitos funcionais e gerenciamento de dados. Esta camada atua como centralizador, possuindo uma única instância para todo o sistema, interagindo diretamente com todas as requisições vindas da Camada Cliente. Além disso, esta camada é a responsável pela sincronização do ambiente colaborativo do $P P O$, que possibilita a colaboração síncrona e assíncrona de vários usuários durante o preenchimento de um planejamento de ensino.

Na Camada Servidor do $P P O$ foi utilizado um conjunto de serviços denominado Backend as a Service (BaaS). BaaS é uma plataforma em nuvem composta pelo servidor de aplicação, banco de dados e uma API, que automatiza o desenvolvimento de backend e cuida de toda infraestrutura necessária para disponibilização de uma aplicação em nuvem [LANE, 2015]. O Firebase ${ }^{2}$ foi a implementação deBaaS utilizada para desenvolvimento da Camada Servidor do PPO.

Firebase é uma plataforma formada por um conjunto de aplicações e serviços em nuvem voltada para o desenvolvimento de aplicativos móveis e aplicativos Web [FIREBASE, 2020]. Em resumo, para construção da Camada Servidor do PPO foi utilizado o

\footnotetext{
${ }^{2}$ https://firebase.google.com
} 
Firebase Authentication para realização da autenticação do usuário via conta do Google. Para armazenamento e sincronização dos dados e gerenciamento do ambiente colaborativo foi utilizado o Cloud Firestore. Por último, foram utilizados o Cloud Functions e FCM para o envio e recebimento de notificações aos usuários na Web.

\subsection{Como utilizar o PBL Planner Online}

Para realização do planejamento o usuário deve autenticar-se com uma conta do Google ou com e-mail e senha e criar um novo planejamento ou abrir algum planejamento já existente para que o sistema apresente a página de Planejamento (Figura 2).

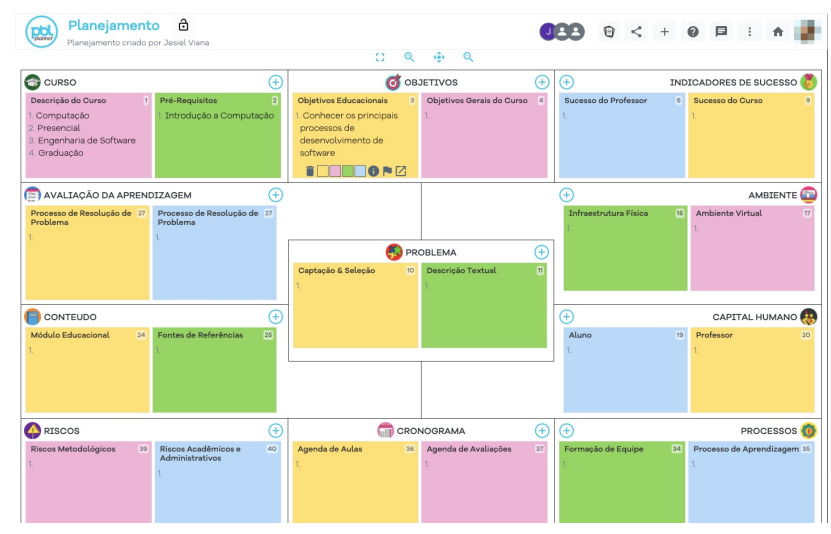

Figura 2. Tela de planejamento

A realização do planejamento é efetuada por meio do preenchimento e organização dos post-its nos campos do PBL Canvas Virtual. Cada campo do PBL Canvas Virtual possui um botão para adicionar um novo post-it, que abre uma modal no formato de post-it para o usuário preenchê-lo de acordo com a carta relacionada. A sequência de preenchimento dos post-its e a disposição em cada campo do canvas é guiado pelas Cartas PBL.

Para facilitar o preenchimento do planejamento guiado pelas Cartas PBL, foi adicionado uma barra lateral com todas as cartas, que pode ser exibida a qualquer momento por meio de um clique no ícone de cartas na barra de navegação.

Qualquer operação realizada no planejamento é sincronizada com todos os colaboradores em tempo real. Da mesma forma, o recebimento das alterações é realizada de forma síncrona, sem a necessidade do usuário recarregar a página. Desse modo, mantémse o planejamento sincronizado em tempo real entre todos os usuários.

A página de planejamento também contempla recursos de percepção, na qual apresenta a listagem de dos colaboradores que estão online e offline, além de destacar os postits em edição. Isso torna a ação de cada usuário mais transparente durante a realização do planejamento, melhorando a experiência do usuário durante a utilização do sistema [FUKS et al., 2011].

Além disso, a barra de menus também possui outros recursos, tais como: chat para facilitar a comunicação entre os colaboradores, exportação do planejamento como plano de ensino e integração com o Trello ${ }^{3}$.

\footnotetext{
${ }^{3}$ https://trello.com/
} 


\section{Avaliação}

Esta seção tem como objetivo apresentar e analisar os resultados obtidos da Avaliação do PPO no segundo Ciclo DSR.

A primeira etapa de avaliação teve como objetivo avaliar a usabilidade da ferramenta. Para isso, foi realizada uma avaliação com especialistas, que contou com a participação de 9 designers, sendo 2 doutores, 5 mestres e 2 especialistas.

Para esta etapa foi utilizado um questionário baseado na escala de usabilidade SUS (System Usability Scale) [BROOKE et al., 1996]. O SUS é formado por 10 questões baseado na escala Likert [LIKERT, 1932].

Segundo Brooke et al. (1996), o resultado do SUS varia de 0 a 100 e é composto pela soma da contribuição individual de cada questão após sua normalização. Para normalização do resultado é aplicada a seguinte fórmula: para as questões ímpares devese subtrair 1 à resposta do usuário, já para as questões pares a pontuação é 5 menos a resposta do usuário. Depois de obter a pontuação de cada item, somam-se os valores e multiplica-se o resultado por 2,5 [BROOKE et al., 1996].

A média final da pontuação obtida dos 9 (nove) avaliadores foi de 73,33. Esse resultado indica que a usabilidade avaliada está acima da média, classificada como "BOA", sendo considerada dentro da escala de aceitabilidade [BANGOR; KORTUM; MILLER, 2008].

Após o feedback dos avaliadores foram realizados alguns ajustes na ferramenta, concluindo assim esta etapa com a aprovação da usabilidade do $P P O$ pelos especialistas. Em seguida uma nova versão do PPO foi disponibilizada para uso em ambientes reais, dando início a etapa de avaliação do uso real do PPO.

Para realização desta etapa foi compartilhado o link do PPO em redes sociais e grupos de Whatsapp para que professores de Computação usassem a ferramenta e participassem voluntariamente de um estudo de caso para avaliação da mesma. Também foi feito o convite para alguns professores que usam PBL para participar da avaliação da ferramenta. Em seguida alguns professores se disponibilizaram para participar dos estudos de caso, utilizando o PPO em um planejamento real.

Estudo de caso é fortemente recomendado por Venable, Pries-Heje e Baskerville (2016), como método de avaliação de pesquisas baseadas em DSR. A recomendação destes autores justifica-se porque o estudo de caso é um método que oferece a possibilidade de avaliar o artefato em um contexto real.

Ao todo foram realizados 3 (três) estudos de caso em 3 (três) Instituições Brasileiras de Ensino Superior (IES), sendo todas três Públicas Federais (IES A, IES B, IES C). 5 (cinco) professores participaram dos estudos de caso, sendo 4 doutores e 1 mestre. 3 (três) professores tinham conhecimento e experiência com PBL e 2 (dois) não tinham nenhuma experiência com a abordagem.

Para execução de todos os estudos de caso, os professores acessaram o $P P O$ por meio do endereço $\mathrm{Web}^{4}$ e em seguida, utilizaram a ferramenta para realização do planejamento guiado somente pelas informações dispostas na própria ferramenta.

\footnotetext{
${ }^{4}$ https://pblplanner.jesielviana.com
} 
Após a execução dos estudos de caso foi realizada a avaliação do PPO por meio de um questionário disponibilizado de forma online para que os professores pudessem responder às perguntas sobre o uso do PPO, logo após eles concluírem o preenchimento do planejamento.

O questionário de avaliação do $P P O$ foi baseado no Shared Workspace Usability Scale - SWUS, que é um modelo de medição para avaliar a interação em sistemas colaborativos proposto por Berkman, Karahoca e Karahoca (2018).

O questionário avaliativo continha 30 (questões), sendo 8 (oito) questões de múltipla escolha sobre o perfil dos participantes, 21 (vinte e uma) questões objetivas, adaptadas do $S W U S$, sobre os recursos de colaboração, automação e satisfação do usuário em relação ao uso da ferramenta, e por fim, 1 (uma) questão aberta para comentários livres. Para as questões objetivas, foi utilizada a escala de Likert com escala de 1 - Discordo Totalmente até 5 - Concordo Totalmente. Todas as questões eram obrigatórias. As respostas objetivas foram agrupadas em 8 (oito) aspectos relacionados ao trabalho colaborativo.

Para facilitar a análise das respostas foi construído o gráfico da Figura 3, que apresenta uma visão geral das respostas obtidas em cada um dos aspectos avaliados.

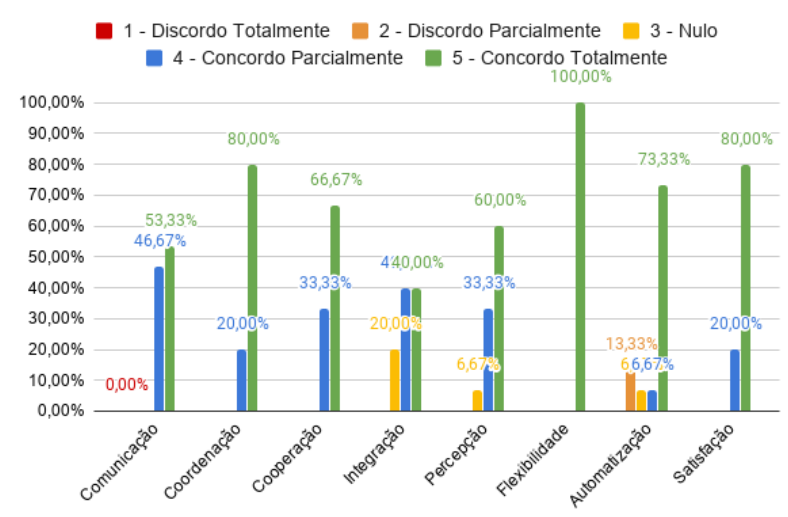

Figura 3. Gráfico dos percentuais obtidos por aspecto relacionado ao SWUS

Neste ciclo, os aspectos relacionados ao Modelo 3C de Colaboração foram avaliados por meio de 7 (sete) perguntas relacionadas as dimensões de comunicação (3), coordenação (1) e cooperação (3).

Sobre a comunicação, $53,33 \%$ das respostas obtidas concordaram totalmente que os recursos disponibilizados pelo $P P O$ atendem as necessidades da colaboração remota e outros $46,67 \%$ das respostas concordando parcialmente, este percentual pode ser motivado pelo uso de outras ferramentas de comunicação durante o planejamento, como foi relatado por alguns professores.

$80 \%$ do professores concordaram totalmente com a forma que a ferramenta faz a coordenação e controle dos usuários e do ambiente virtual compartilhado.

Já sobre a cooperação, $66,67 \%$ das respostas obtidas concordaram totalmente com a forma que o PPO possibilita a realização do planejamento colaborativo em grupo por meio do seu ambiente virtal compartilhado. Outros 33,33\% concordaram parcialmente. A fim de entender quais as carências e problemas da ferramenta, foi realizado entrevista 
com os professores que concordaram de forma parcial e foram identificadas sugestões de ajustes no ambiente compartilhado para colaboração assíncrona, tais como: melhoria na identificação das colaborações realizadas por cada usuário e criação do histórico de alterações do planejamento.

A Integração foi avaliada por meio 1 (uma) questão que tinha por objetivo verificar se utilizando o $P P O$ os participantes poderiam compreender as intenções de outros usuários e chegarem a um consenso sobre o planejamento a ser realizado. As respostas para concordância total foi $40,40 \%$, concordância parcial $40,00 \%$ e $20 \%$ responderam "Nulo". Em entrevista com os avaliadores foi identificada necessidade de melhoria quanto à formulação da pergunta.

Sobre o aspecto Percepção, 3 (três) questões foram utilizadas na medição, que avaliaram sobre a ciência que o usuário tem de que faz parte de um grupo (Q20), ciência das ações dos outros usuários online $(\mathrm{Q} 21)$ e entendimento sobre suas ações $(\mathrm{Q} 22)$. Ao todo, $60,00 \%$ das respostas concordaram totalmente com os recursos de percepção disponíveis na ferramenta e outros 33,33\% concordaram parcialmente. Segundo os avaliadores, há necessidade de alguns ajustes para melhorar a percepção durante a colaboração assíncrona. Já na colaboração síncrona, todos os avaliadores mostraram-se satisfeitos com os recursos disponibilizados pela ferramenta.

A Flexibilidade promovida pela ferramenta foi avaliada por meio de 2 (duas) questões, em que $100 \%$ das respostas obtidas concordaram totalmente que oPPO promove a flexibilidade de uso em relação ao tempo e local para realização do planejamento colaborativo, além de facilitar o compartilhamento entre educadores.

Em relação ao aspecto de Automatização, 73,33\% das respostas obtidas concordaram totalmente que as funcionalidades de geração automática de artefatos e integração com ferramentas de gestão de tarefas é útil para formalização de documentos junto a gestão educacional e facilita o acompanhamento e controle do planejamento por meio de ferramentas específicas. O acompanhamento das tarefas planejadas é um fator importante para o sucesso do método PBL em um curso ou disciplina [SANTOS et al., 2018].

A satisfação do usuário foi avaliada por meio de 3 (três questões), que verificou a simplicidade $(\mathrm{Q} 27)$ e prazer dos professores em utilizar o $P P O(\mathrm{Q} 28)$ e se eles indicariam o PPO para outros professores (Q29). As respostas para concordância total foram $80,00 \%$ e, 20,00\% com concordância parcial. Este item indica que na concepção dos professores, o $P P O$ atende às necessidades do planejamento colaborativo na abordagem PBL para cursos de Computação. Outro ponto a se destacar, é que alguns professores relataram em entrevista, que o PPO promove e motiva a realização do planejamento, que muitas vezes é negligenciado pelos docentes.

Por fim, através das questões abertas do questionário e de entrevistas com os avaliadores foram coletados relatos sobre a percepção dos professores ao utilizar o PPO. A seguir serão descritos alguns desses relatos. R1: "O plano de ensino gerado a partir do canvas é muito útil pois mostra com detalhes o que foi pensado para a disciplina, além de proporcionar melhorias com relação a metodologia e atividades realizadas". R2: "O PBL Planner Online promove o compartilhamento e organização de informações entre a equipe". R3: "É uma ferramenta muito útil para divulgar/promover entre professores o método PBL". R4: "A utilização da ferramenta é ótima, pois me obriga a fazer um planeja- 
mento detalhado, incluindo aspectos importantes relacionados ao ensino e aprendizagem.

Os resultados apresentados acima indicam uma boa aceitação e utilidade do PPO para elaboração do planejamento colaborativo de ensino PBL, uma vez que por meio de seu ambiente virtual, estruturado com aspectos relevantes do planejamento PBL, guias e processo baseado nos princípios PBL, possibilitam aos docentes planejar as atividades de forma consistente e colaborativa de forma síncrona e assíncrona.

Entre os principais benefícios citados pelos professores que utilizaram a ferramenta, vale destacar o processo de realização do planejamento guiado pelos princípios PBL orientado à colaboração. O processo guia de forma ordenada o planejamento de todos os elementos importantes para realização de um curso na abordagem PBL.

Outro fator a se destacar é a possibilidade da colaboração distribuída assíncrona proporcionada pelo PPO. Esse recurso foi bastante utilizado durante as realizações dos planejamentos e foi fundamental para a realização dos estudos de casos, visto que durante todo o ano de 2020 todas as aulas e consequentemente os planejamentos foram remotos devido a pandemia provocada pela COVID-19 [ARRUDA, 2020].

\section{Conclusão}

Uma vez que o planejamento educacional na abordagem PBL deve ser realizado com a participação de todos os envolvidos, mas nem sempre é possível uma reunião presencial, é importante a utilização de uma ferramenta que possa romper as barreiras impostas pelo espaço físico e o tempo, e assim, facilitar a colaboração de forma coordenada.

Com objetivo de possibilitar a colaboração distribuída entre educadores durante a realização do planejamento de ensino na abordagem PBL foi criado o PBL Planner Online $(P P O)$. A relevância da proposição de uma ferramenta colaborativa para apoiar o planejamento do ensino PBL reside na falta de ferramentas específicas para esse fim, especialmente que atenda aos princípios da abordagem PBL.

Os resultados das avaliações do $P P O$ mostraram indicadores positivos em relação à utilização da ferramenta para o planejamento colaborativo do ensino na abordagem PBL com membros distribuídos. Como ponto de melhoria, as avaliações indicam ajustes em relação à percepção durante o planejamento assíncrono.

Por fim, o PPO possibilitou o Trabalho Colaborativo Remoto realizado de forma síncrono e assíncrono, tornando a colaboração durante a realização do planejamento mais flexível. Nesse sentido, a contribuição dada pelo $P P O$ foi reconhecida pelos professores que participaram da avaliação.

Como trabalhos futuros, pretende-se realizar melhorias de usabilidade, implementar um processo de revisão automática do planejamento e executar um novo ciclo $D S R$ para avaliação do $P P O$ em outras instituições de ensino.

\section{Referências}

ALEXANDRE, G. H. et al. Applying and managing pbl-an experience in information systems education. In: CSEDU (2). [S.1.: s.n.], 2018. p. 57-67. 
ALEXANDRE, G. H. d. S. PBL PLANNER TOOLKIT: Uma ferramenta para o planejamento da abordagem PBL no ensino de Computação. Tese (Doutorado) Universidade Federal de Pernambuco, Recife, 2018.

ARRUDA, E. P. Educação remota emergencial: elementos para políticas públicas na educação brasileira em tempos de covid-19. EmRede-Revista de Educação a Distância, v. 7, n. 1, p. 257-275, 2020.

ASSIS, M. P. de; ALMEIDA, M. E. B. de. Learning design e tecnologias: criação de ambientes colaborativos para a aprendizagem. Psicologia da Educação. Programa de Estudos Pós-Graduados em Educação: Psicologia daf Educação. ISSN 2175-3520, n. 44, 2017.

BANGOR, A.; KORTUM, P. T.; MILLER, J. T. An empirical evaluation of the system usability scale. Intl. Journal of Human-Computer Interaction, Taylor \& Francis, v. 24, n. 6, p. 574-594, 2008.

BANIYA, S. et al. Creating interdisciplinary collaborative teaching/learning praxis with design thinking, communication, and composition. In: Proceedings of the 37th ACM International Conference on the Design of Communication. [S.1.: s.n.], 2019. p. 1-6.

BARBOSA, D. M.; BAX, M. A design science como metodologia para a criação de um modelo de gestão da informação para o contexto da avaliação de cursos de graduação. Revista Ibero-Americana De Ciência Da Informação, v. 10, n. 1, p. 32-48, 2017.

BERKMAN, M. I.; KARAHOCA, D.; KARAHOCA, A. A measurement and structural model for usability evaluation of shared workspace groupware. International Journal of Human-Computer Interaction, Taylor \& Francis, v. 34, n. 1, p. 35-56, 2018.

BERTOCCO, M. et al. A client-server architecture for distributed measurement systems. IEEE transactions on instrumentation and measurement, IEEE, v. 47, n. 5, p. 1143-1148, 1998.

BROOKE, J. et al. Sus-a quick and dirty usability scale. Usability evaluation in industry, London-, v. 189, n. 194, p. 4-7, 1996.

BROUGHTON, M. et al. Being here: Designing for distributed hands-on collaboration in blended interaction spaces. In: . New York, NY, USA: ACM, 2009. (OZCHI '09), p. 73-80. ISBN 9781605588544.

BROWN, S. The C4 model for software architecture. 2018.

ELLIS, C. A.; GIBBS, S. J.; REIN, G. Groupware: some issues and experiences. Communications of the ACM, ACM New York, NY, USA, v. 34, n. 1, p. 39-58, 1991.

FIREBASE. Firebase. [S.1.], 2020. [Online]. Available:〈https://firebase.google.com〉. Accessed on July 20, 2020.

FUKS, H. et al. Do modelo de colaboração 3c à engenharia de groupware. Simpósio Brasileiro de Sistemas Multimídia e Web-Webmidia, p. 0-8, 2003.

FUKS, H. et al. Applying the $3 C$ model to groupware engineering. [S.1.]: PUC Rio de Janeiro, 2004.

FUKS, H. et al. Teorias e modelos de colaboração. Sistemas colaborativos, Elsevier, p. 16-33, 2011. 
GOMES, R. L.; WILLRICH, R.; RIVERA, G. Arquiteturas distribuídas para sistemas colaborativos. Sistemas Colaborativos. Rio de Janeiro: Elsevier, p. 328-346, 2011.

GOMIDES, M. D. A. et al. Aprendizagem baseada em problemas na formação médica: uma revisão integrativa da literatura. Revista EDaPECI, v. 19, n. 3, p. 27-41, 2019.

LANE, K. Overview of the backend as a service (baas) space. API Evangelist, 2015.

LIKERT, R. A technique for the measurement of attitudes. Archives of psychology, 1932.

OLIVEIRA, A. M. C. A.; SANTOS, S. C. dos; GARCIA, V. C. Pbl in teaching computing: An overview of the last 15 years. In: IEEE. 2013 IEEE Frontiers in Education Conference (FIE). [S.1.], 2013. p. 267-272.

PIMENTEL, M. et al. Modelo 3c de colaboração para o desenvolvimento de sistemas colaborativos. Anais do III Simpósio Brasileiro de Sistemas Colaborativos, p. 58-67, 2006.

RAMA, J.; BISHOP, J. A survey and comparison of cscw groupware applications. In: . [S.l.]: South African Institute for Computer Scientists and Information Technologists, 2006. (SAICSIT '06), p. 198-205. ISBN 1595935673.

RODRIGUES, A. N.; SANTOS, S. C. dos. A framework for applying problem-based learning to computing education. In: IEEE. 2016 IEEE Frontiers in Education Conference (FIE). [S.1.], 2016. p. 1-7.

SANTOS, S. C. D. Pbl-see: An authentic assessment model for pbl-based software engineering education. IEEE Transactions on Education, IEEE, v. 60, n. 2, p. 120-126, 2016.

SANTOS, S. C. dos et al. How to apply problem-based learning in a managed way? a case in computing education. In: SPRINGER. International Conference on Computer Supported Education. [S.1.], 2018. p. 287-309.

SANTOS, S. C. dos; FIGUERÊDO, C. O.; WANDERLEY, F. Pbl-test: A model to evaluate the maturity of teaching processes in a pbl approach. In: IEEE. 2013 IEEE Frontiers in Education Conference (FIE). [S.1.], 2013. p. 595-601.

SANTOS, S. C. dos; FURTADO, F.; LINS, W. xpbl: A methodology for managing pbl when teaching computing. In: IEEE. 2014 IEEE Frontiers in Education Conference (FIE) Proceedings. [S.1.], 2014. p. 1-8.

SAVERY, J. R. Overview of problem-based learning: Definitions and distinctions. Essential readings in problem-based learning: Exploring and extending the legacy of Howard S. Barrows, v. 9, p. 5-15, 2015.

VENABLE, J.; PRIES-HEJE, J.; BASKERVILLE, R. Feds: a framework for evaluation in design science research. European journal of information systems, Taylor \& Francis, v. 25, n. 1, p. 77-89, 2016.

VIDAL, T. C.; SANTOS, S. C. D.; CARVALHO, R. S. Pbl-tutor canvas: A tool based on backward design to plan pbl in computing education. In: IEEE. 2016 IEEE Frontiers in Education Conference (FIE). [S.1.], 2016. p. 1-8.

WIERINGA, R. J. Design science methodology for information systems and software engineering. [S.1.]: Springer, 2014. 